\title{
Rubella Immune Status among Infertile Women: A Priority Vaccination Cohort
}

\author{
Prasanna L Kakarla ${ }^{1}$, Anil K Bilolikar², Sukrutha G Reddy ${ }^{3}$, Vyjayanthi Sreenivasan ${ }^{4}$
}

\begin{abstract}
Aim and objective: Congenital rubella syndrome (CRS), a consequence of rubella virus infection in the first trimester of pregnancy is a silent crippling disease endemic in India. This study sought to find out and vaccinate rubella susceptible women attending a fertility clinic in an urban setup.

Materials and methods: As an institutional policy, all females attending fertility clinic were screened for anti-rubella lgG antibodies. A total 2272 non-duplicate serum samples received between January 2011 and December 2017 were processed using enzyme-linked fluorescent assay using mini VIDAS system following standard recommendations. Any value $<10 \mathrm{IU} / \mathrm{mL}$ was regarded negative, between 10 and 15 as equivocal and $>15$ as positive.

Results: An average of $10.95 \%$ women was susceptible to rubella whereas $86.75 \%$ were immune to it. Samples from 52 women gave equivocal results and they were considered to be susceptible. All susceptible females were vaccinated with R-vac and treated for infertility one month after vaccination. Age-specific immune status was not significant. A trend analysis revealed steady increase in immunity from 2011 to 2017 with a slight drop in 2016.

Conclusion: The proportion of susceptible females of reproductive age who need to be vaccinated is significant. This study emphasizes the need for preconception screening as we would have an opportunity to vaccinate susceptible women.

Clinical significance: There is a need for preconception screening of rubella not only in infertile women but also normal reproductive age females. With successful eradication of polio, elimination of rubella and measles being the next targets, combined vaccination strategy is the key to rubella elimination.

Keywords: Age-specific immunity, Congenital rubella syndrome, Laboratory research, MR vaccine, Preconception screening, Trend. International Journal of Infertility and Fetal Medicine (2021): 10.5005/jp-journals-10016-1227
\end{abstract}

\section{INTRODUCTION}

Rubella virus, the causative agent of the mildest of common viral exanthems, is concerning due to the consequences if acquired in utero. ${ }^{1}$ An infection during early weeks of pregnancy leads to congenital rubella syndrome (CRS), which is a silent crippling disease endemic in India. More than one lakh cases of CRS may occur each year in developing countries alone. ${ }^{2}$ The manifestations of CRS in a child are multitude and the most common being Gregg's triad (Cataracts, Cardiovascular defects and Deafness). Various studies attribute $10-15 \%$ of intrauterine infections to rubella and $1-60 \%$ of ocular abnormalities, $1.5-29 \%$ of congenital deafness, $30 \%$ of congenital heart disease to CRS. ${ }^{3}$ Rubella infection can also result in fetal death, still birth and spontaneous abortion.

Infertility is an underestimated problem world over. WHO estimates that one in every four couples in developing countries is affected by infertility. ${ }^{4}$ Based on the census reports of India, childlessness has risen by $50 \%$ since $1981 .{ }^{5}$ This translates into a huge number considering the population of India. Infertile women are considered to be the priority vaccination cohort, as any rubella infection in their subsequent pregnancy causes substantial physiological, psychological and economic burden on parents. Hence there are established protocols to investigate the rubella immune status of all infertile women before treating them.

Subclinical nature of infection and non-descript rash negate the reliability of history of infection as an index of rubella immunity. Demonstration of anti-rubella IgG antibodies by immunoassays is reliable as there is only one serotype of virus. ${ }^{6}$
${ }^{1}$ Department of Microbiology, Sri Venkateswara Medical College, Tirupati, Andhra Pradesh, India

2,3 Department of Microbiology, Krishna Institute of Medical Sciences Limited, Secunderabad, Telangana, India

${ }^{4}$ Infertility Clinic, Department of Obstetrics and Gynaecology, Krishna Institute of Medical Sciences Limited, Secunderabad, Telangana, India

Corresponding Author: Prasanna L Kakarla, Department of Microbiology, Sri Venkateswara Medical College, Tirupati, Andhra Pradesh, India, Phone: +91 8332055939, e-mail: prasannakakarla380@ gmail.com

How to cite this article: Kakarla PL, Bilolikar AK, Reddy SG, et al. Rubella Immune Status among Infertile Women: A Priority Vaccination Cohort. Int J Infertil Fetal Med 2021;12(3):55-59.

Source of support: Nil

Conflict of interest: None

There are no national estimates of risk of acquiring rubella among childbearing age females in India. With no national screening policy in place, it is difficult to gather that data. Screening of infertile women, a large subset of reproductive age females, might reflect the immunity to rubella in this age group. India has introduced the measles-rubella (MR) vaccine in five states and union territories (Karnataka, Tamil Nadu, Puducherry, Goa and Lakshadweep) in February 2017. ${ }^{7}$ The timeline of introduction of rubella vaccine is tabulated below as Table $1 .^{8-10}$ Monitoring ageand sex-specific seroprevalence provides data for identification of necessary modifications to immunization strategy. 
Table 1: Summary of timeline of rubella vaccination programs in India

\begin{tabular}{lllll}
\hline Year & Dose & Age and sex & Vaccine strain & Comments \\
\hline 1999 & 1 & $15-18$ months & MMR & State immunization program of Delhi \\
2006 & 2 & $15-18$ months; 4-6 years & MMR & Recommended by IAP for affordable parents \\
2017 & 1 & 9 months-15 years & MR & Launched in 5 states (Karnataka, Tamil Nadu, \\
& 1 & 9 months-15 years & & Puducherry, Goa and Lakshadweep) \\
Next 18 months & 2 & $9-12$ months; 16-24 months & MR & All over India in 4 phases \\
2018 & & Universal Immunization Program
\end{tabular}

MMR, measles-mumps-rubella; IAP, Indian Academy of Pediatrics

This study sought to find out and vaccinate rubella susceptible women among those attending a fertility clinic in an urban setup. This study also aimed to identify age-specific immune status of this cohort and to find out the trend of rubella immunity among infertile females between January 2011 and December 2017.

\section{Materials and Methods}

\section{Study Design}

Retrospective and prospective laboratory-based observational cross-sectional study. The data was collected retrospectively using laboratory information management system from 2011 to 2015, and prospectively during 2016 and 2017.

\section{Study Duration}

January 1, 2011 to December 31, 2017.

\section{Ethical and Human Considerations}

All the procedures followed were in accordance of the ethical standards laid down by the ICMR's Ethical guidelines for biomedical and health research on human participants (2017). The study got clearance from institutional ethics committee. The study subjects were explained the purpose of the study and assured confidentiality. Written informed consent was taken before sample collection for prospective subjects.

\section{Study Population}

All female patients attending the fertility clinic at the study Hospital.

\section{Inclusion Criteria}

Female patients of reproductive age with a primary complaint of infertility attending the fertility clinic were included in the study. Clinical definition of infertility, as given by $\mathrm{WHO}$, as "a disease of reproductive system defined by the failure to achieve a clinical pregnancy after 12 months or more of regular unprotected sexual intercourse" was used in the study. ${ }^{11}$ Reproductive age was considered to be $15-49$ years.

\section{Exclusion Criteria}

Nil

\section{Sampling}

All consecutive, non-duplicate samples were included during the study period.

\section{Sample Processing}

A total of 2272 non-duplicate serum samples were received for testing during the study period. They were processed using enzyme-linked fluorescent assay using mini VIDAS system (bioMerieux, France) following manufacturers' recommendations. ${ }^{12}$ This assay combines a 2-step enzyme immunoassay sandwich method with a final fluorescent detection. The entire reaction was carried out in a solid phase receptacle (SPR) the interior of which was coated with rubella virus antigen. The intensity of fluorescence detected was proportional to the concentration of antibodies present in the sample. Fluorescence was calculated twice for each sample tested. The first reading was a background reading of the substrate cuvette and the second reading was taken after incubating the substrate with enzyme remaining on the interior of the SPR. The relative fluorescence value (RFV) was calculated by subtracting background reading from the final result.

At the end of the assay, the results were calculated by the instrument in relation to the calibration curve (4-parameter logistic model) stored in the memory and were expressed in $\mathrm{IU} / \mathrm{mL}$ as per the WHO standard. Any value $<10$ was regarded negative, between 10 and 15 as equivocal and $>15$ as positive. Quality control of the test and calibration of the instrument were performed with each new kit or every 14 days, whichever is earlier.

\section{Statistical Evaluation}

Data was statistically analyzed using SPSS version 20 (IBM Inc., SPSS Inc., Chicago, IL, USA) software and results were presented through suitable tables and graphs. Continuous variables were summarized using descriptive statistics in terms of means \pm standard deviations and $95 \%$ confidence intervals $(95 \% \mathrm{Cl})$. $p$ values $<0.05$ were considered statistically significant.

\section{Results and Discussion}

\section{Baseline Characteristics}

Mean age (SD) of the patients was 30.47 years (5.25). Age range of the patients studied was 17-49 years. Mean age of females was higher when compared to other studies as the study group comprised of infertile females.

\section{Rubella Seropositivity Characteristics}

Out of 2272 serum samples received, anti-rubella IgG positive, equivocal and negative results were observed in $86.75 \%, 2.28 \%$ and $10.95 \%$ of samples respectively. Samples from 52 (2.28\%) women gave equivocal results and they were considered to be susceptible. An average of $11.08 \%$ women was susceptible to rubella whereas $86.75 \%$ were immune to it. This implies that one in 10 females tested was susceptible to rubella, which is high.

The percentage susceptibility in this study $(10.95 \%)$ is comparable to the result from the National Institute of Communicable Diseases which showed $12 \%$ overall susceptibility to rubella amongst pregnant women in Delhi from 1988 to $2002 .^{13}$ These results are also in par with few studies conducted in adolescent and adult females in India. ${ }^{14}$ However, it is higher when compared to many other studies with adolescents or adult females as study subjects. ${ }^{15-17}$

Rates of rubella immunity have been variable in different parts of the world. The current results compare with results from Jordan 
(90.9\%), Kuwait (92.3\%), Oman (92\%), Iran (94.6\%), the United States (91\%), and Canada (91.8\%), and seropositivity in the current research is greater than the percentages in Sri Lanka (76\%) and Nigeria (68.5\%). ${ }^{18-22}$ However, all these results were from either antenatal females or adolescent girls.

Age-specific seroprevalence among girls, according to $\mathrm{WHO}$, is a sensitive tool for risk assessment of CRS. ${ }^{2}$ An analysis of age-specific immunity in the current study yielded results as shown in Figure 1.

Highest immunity was noted in 45-50 years females followed by $21-25$ years women. However this difference was not statistically significant $(p=0.45)$. Equivocal results were not observed in the extreme ages; the results were either clear negatives or clear positives. Four (23.52\%) out of seventeen were rubella non-immune in the 15-20 years group. This higher susceptibility observed among 15-20 years age group could be due to undeveloped immunity. Similar results were obtained in as study conducted in Jordan. ${ }^{18}$ An improving trend with age in the rubella immune status was not evident in the present study (Table 2 ) as the age group studied comprised of adult females and not girls. In a study done in Saudi Arabia, there was a significant decrease in immunity with age ( $p=$ 0.0005). This difference could be due to the robust immunization program involving children. ${ }^{23}$

\section{Seropositivity Trend}

The total number of samples received and their year-wise split is given as Table 3.

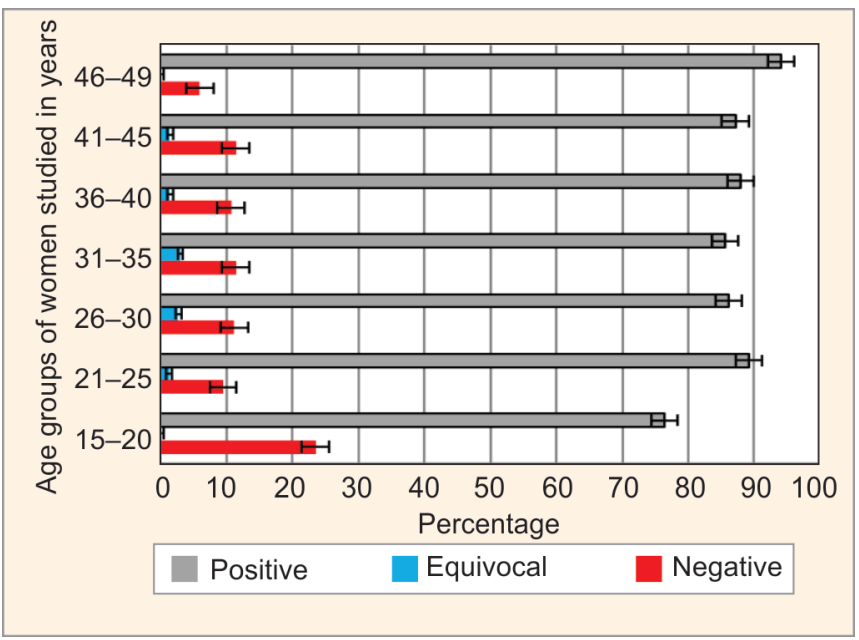

Fig. 1: Age-specific immune status against rubella depicted along with standard error
There was an increasing trend in seropositivity from $83.69 \%$ in 2011 to $88.6 \%$ in 2017 with a slight drop in the year 2016. A trend analysis revealed steady increase in immunity from 2011 to 2017, with a slight drop in the year 2016. This can be attributed to higher usage of MMR vaccine owing to increasing awareness in the recent past. ${ }^{6}$ Even with the increasing immunity, a substantial proportion of women were susceptible to rubella calling for a robust program.

The trend of rubella seropositivity is depicted as a line diagram in Figure 2. A cumulative trend is also depicted in the same graph assuming that all females who were seropositive remain so during the study period.

\section{Follow-up}

All susceptible females (whose samples yielded either negative or equivocal results) were vaccinated with R-vac (Serum Institute of India Ltd) and treated for infertility one month after vaccination. Testing for seroconversion post-vaccination was not attempted as the vaccine is effective. ${ }^{24}$

\section{Prevention of CRS}

There are two strategies to prevent CRS: CRS reduction and rubella/CRS elimination. The direct strategy aims to vaccinate adolescent girls to prevent infection among them, but cannot prevent transmission of rubella virus. The indirect strategy aims to immunize children before they are naturally exposed to infection and interrupts rubella virus circulation. However, it might increase the probability of rubella outbreaks among adult population and CRS as occurred in Greece. ${ }^{25,26}$ The implementation of either strategy is dependent on many issues, such as infrastructure, goals of the program, and funding. ${ }^{27}$

According to Plotkin, vaccination of all infants will probably eradicate CRS in 30-40 years, vaccination of all schoolgirls will presumably eradicate CRS in 10-20 years, and vaccination of adult women will eradicate CRS immediately, but only if $100 \%$ are immunized. ${ }^{28}$

Countries that have not introduced rubella vaccination may introduce it along with the accelerated measles control and elimination (according to updated WHO rubella vaccine recommendations). ${ }^{29}$ The preferred strategy for this is to initiate a campaign with MR/MMR vaccine targeting a wide range of ages and immediate introduction of MR/MMR vaccine into the national immunization program. As a welcome sign, Government of India has taken initiative to give MR vaccine to all children aged 9 months to 15 years, starting with 5 states. Expanding the provision of MR vaccine to other states and continued surveillance of vaccination are key steps to achieve elimination of measles and control of rubella by 2020 .

Table 2: Immunity rate to rubella as a function of age in infertile females in South India

\begin{tabular}{lccll}
\hline Age group (Years) & $\begin{array}{l}\text { Total number } \\
\text { studied }\end{array}$ & $\begin{array}{l}\text { Total number } \\
\text { positive* }\end{array}$ & Immune women \% (95\% Cl) & $\chi^{2}(p)$ \\
\hline $15-20$ & 17 & 13 & $76.47(56.30-96.63 \%)$ & $5.71(0.45)$ \\
$21-25$ & 380 & 339 & $89.21(86.09-92.32 \%)$ & \\
$26-30$ & 870 & 750 & $86.2(83.91-88.49 \%)$ \\
$31-35$ & 637 & 545 & $85.55(82.82-88.28 \%)$ \\
$36-40$ & 281 & 247 & $87.9(84.08-91.71 \%)$ \\
$41-45$ & 70 & 61 & $87.14(79.30-94.98 \%)$ \\
$46-49$ & 17 & 16 & $94.11(82.93-105.30 \%)$ \\
Total & 2272 & 1971 & $86.75(85.35-88.14 \%)$ & \\
\hline
\end{tabular}

$95 \% \mathrm{Cl}, 95 \%$ confidence interval; $\chi^{2}$, Chi-square test; $p, p$ value

*Note: All equivocal samples were considered to be negatives for the above statistical calculation 
Rubella Immune Status among Infertile Women: A Priority Vaccination Cohort

Table 3: Year-wise distribution of positive, equivocal and negative samples studied

\begin{tabular}{llllll}
\hline S. no. & Year & $\begin{array}{l}\text { Samples tested positive } \\
(>15 \mathrm{IU} / \mathrm{mL})(n=1971)\end{array}$ & $\begin{array}{l}\text { Samples tested equivocal } \\
(10-15 \mathrm{IU} / \mathrm{mL})(n=52)\end{array}$ & $\begin{array}{l}\text { Samples tested negative } \\
(<10 / \mathrm{J} / \mathrm{mL})(n=249)\end{array}$ & $\begin{array}{l}\text { Total number of samples } \\
\text { studied }(n=2272)\end{array}$ \\
\hline 1 & 2011 & $231(83.69 \%)$ & $8(2.89 \%)$ & $37(13.40 \%)$ & 276 \\
2 & 2012 & $278(85.01 \%)$ & $5(1.52 \%)$ & $44(13.45 \%)$ & 327 \\
3 & 2013 & $305(87.14 \%)$ & $13(3.71 \%)$ & $32(9.14 \%)$ & 350 \\
4 & 2014 & $341(87.66 \%)$ & $7(1.79 \%)$ & $41(10.53 \%)$ & 389 \\
5 & 2015 & $262(87.91 \%)$ & $6(2.01 \%)$ & $30(10.06 \%)$ & 298 \\
6 & 2016 & $243(86.41 \%)$ & $4(1.42 \%)$ & $34(12.09 \%)$ & 281 \\
7 & 2017 & $311(88.60 \%)$ & $9(2.56 \%)$ & $31(8.83 \%)$ & 351 \\
\hline
\end{tabular}

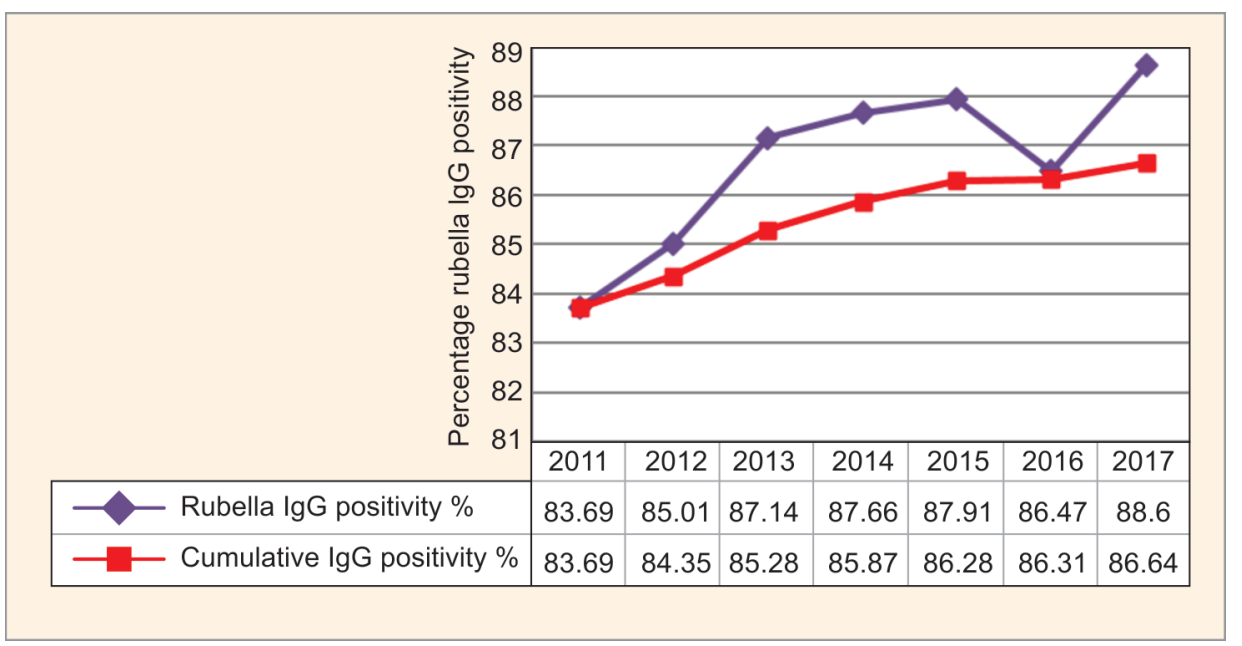

Fig. 2: Trend of rubella IgG positivity among reproductive age group women attending infertility clinic

\section{Strengths and Limitations of Study}

This study is novel with respect to study subjects being infertile, non-pregnant women. Though this data might not truly represent rubella immunity among females in the community, it gives an estimate of the susceptibility levels. Data from our hospital was not available to compare with susceptibility among antenatal/ adolescent females.

\section{Conclusion}

This study emphasizes the need for preconception screening as we would have an opportunity to vaccinate susceptible women. Agespecific immunity in this population cohort is not very informative and requires further studies. Though improving immunity to rubella year by year is a welcome sign, still the proportion of susceptible females of reproductive age who need to be vaccinated is significant. With successful eradication of polio, elimination of rubella and measles being the next targets, combined vaccination strategy is the key to rubella elimination.

\section{Acknowledgments}

The author would like to thank the whole Department of Microbiology and Infertility Clinic, Krishna Institute of Medical Sciences Ltd for their support.

\section{Manufacturer Name}

Mini VIDAS system (bioMerieux, Marcy-I'Etoile, France).

\section{References}

1. Grangeot-Keros L. Rubella and pregnancy. Pathol-Biol 1992;40(7):706710.

2. WHO. Rubella vaccines position paper. Wkly Epidemiol Rec 2000;20:161-172.

3. Dewan P, Gupta P. Burden of congenital rubella syndrome (CRS) in India: a systematic review. Indian Pediatr 2012;49(5):377-399. DOI: 10.1007/s13312-012-0087-4.

4. Mascarenhas MN, Flaxman SR, Boerma T, et al. National, regional, and global trends in infertility prevalence since 1990: a systematic analysis of 277 health surveys. PLoS Med 2012;9(12):e1001356. DOI: 10.1371/journal.pmed.1001356.

5. Ganguly S, Unisa S. Trends of infertility and childlessness in India: findings from NFHS sata. Facts Views Vis Obgyn 2010;2(2): 131-138.

6. Lambert N, Strebel P, Orenstein W, et al. Rubella. Lancet 2015;385(9984):2297-2307. DOI: 10.1016/S0140-6736(14) 60539-0.

7. Ghosh A, Measles-Rubella Vaccine to Roll Out in February. The Indian Express; 10 January, 2017. Available from: http://www. indianexpress.com/article/india/measles-rubella-vaccine-to-rollout-in -february-4467248/. [Last cited on 2017 Dec 14].

8. Gomber S, Arora SK, Das S, et al. Immune response to second dose of MMR vaccine in Indian children. Indian J Med Res 2011;134: 302-306.

9. Singhal T, Amdekar YK, Agarwal RK, ed. IAP Guidebook on Immunisation, IAP Committee on Immunization 2007-2008. New Delhi: Jaypee Brothers Medical Publishers; 2009. p. 16-98.

10. Singh S. Indian Academy of Paediatrics Committee on Immunization. Update on immunization policies, guidelines and recommendations. Indian Pediatr 2004;41(3):240-242. 
11. Zegers-Hochschild F, Adamson GD, de Mouzon J, et al. The International Committee for Monitoring Assisted Reproductive Technology (ICMART) and the World Health Organization (WHO) revised glossary on ART terminology, 2009. Hum Reprod 2009;24(11):2683-2687. DOI: 10.1093/humrep/dep343.

12. VIDAS RUB IgG II (RBG) [Package Insert]. Marcy-I'Etoile-France: bioMerieux SA; 2016.

13. Gandhoke I, Aggarwal R, Lal S, et al. Seroprevalence and incidence of rubella in and around Delhi (1988-2002). Indian J Med Microbiol 2005;23(3):164-167. DOI: 10.4103/0255-0857.16587.

14. Gohil DJ, Kothari ST, Chaudhari AB, et al. Seroprevalence of measles, mumps, and rubella antibodies in college students in Mumbai, India. Viral Immunol 2016;29(3):159-163. DOI: 10.1089/vim.2015.0070.

15. Sharma H, Chowdhari S, Raina TR, et al. Sero-surveillance to assess immunity to rubella and assessment of immunogenicity and safety of a single dose of rubella vaccine in school girls. Indian J Community Med 2010;35(1):134-137. DOI: 10.4103/0970-0218.62575.

16. Phalgune DS, Yervadekar RC, Sharma HJ, et al. Sero-surveillance to assess rubella susceptibility and assessment of immunogenicity and reactogenicity of rubella vaccine in Indian girls aged 18-24 years. Hum Vaccin Immunother 2014;10(10):2813-2818. DOI: 10.4161/21645515.2014.970947.

17. Singla N, Jindal N, Aggarwal A. The seroepidemiology of rubella in Amritsar (Punjab). Indian J Med Microbiol 2004;22(1):61-63. DOI: 10.1016/S0255-0857(21)02955-8.

18. Jarour N, Hayajneh WA, Balbeesi A, et al. Seroprevalence of rubella among jordanian women of childbearing age. Vaccine 2007;25(18):3615-3618. DOI: 10.1016/j.vaccine.2007.01.058

19. Makhseed M, Moussa MA, Ahmed MA, et al. The status of rubella immunity among pregnant women in Kuwait: screening in childbearing age should be reintroduced. Acta Trop 2001;78(1):35-40. DOI: 10.1016/s0001-706x(00)00163-7.
20. Ganjooie TA, Mohammadi MM. The prevalence of antibodies against rubella in pregnant women in Kerman, Iran. Saudi Med J 2003;24(11):1270-1271.

21. Haas DM, Flowers CA, Congdon CL. Rubella, rubeola, and mumps in pregnant women: susceptibilities and strategies for testing and vaccinating. Obstetr Gynecol 2005;106(2):295-300. DOI: 10.1097/01. AOG.0000171110.49973.e3.

22. Kaushal M, Baxi A. Rubella immune status of pregnant \& non-pregnant women in Indian population. Int J Gynecol Obstet 2007;6(2):1-6.

23. Alsibiani SA. Rubella immunity among pregnant women in Jeddah, Western Region of Saudi Arabia. Obstet Gynecol Int 2014;2014:659838. Available from: 10.1155/2014/659838.

24. Matter L, Kogelschatz K, Germann D. Serum levels of rubella virus antibodies indicating immunity: response to vaccination of subjects with low or undetectable antibody concentrations. J Infect Dis 1997;175(4):749-755. DOI: 10.1086/513967.

25. Grant GB, Reef SE, Dabbagh A, et al. Rubella and congenital Rubella syndrome control and elimination - Global progress, 2000-2014, WHO. Wkly Epidemiol Rec 2015;90:510-516.

26. Panagiotopoulos T, Antoniadou I, Valassi-Adam E. Increase in congenital rubella occurrence after immunization in Greece: retrospective survey and systematic review. BMJ 1999;319(7223):14621467. DOI: $10.1136 / \mathrm{bmj} .319 .7223 .1462$.

27. Vardas E, Lancet Laboratory Newsletter [Internet]. 2011 [Cited 2018 Apr 8]. Available from: http://secure.lancet.co.za/files/1813/2257/6116/ RubellaNewsletterNov2011.pdf.

28. Robertson SE, Cutts FT, Samuel R, et al. Control of rubella and congenital rubella syndrome (CRS) in developing countries, part 2: vaccination against rubella. Bullet World Health Organizat 1997;75(1):69-80.

29. WHO Publication. Rubella vaccines: WHO position paperrecommendations. Vaccine 2011;29(48):8767-8768. 